\title{
Evaluation of Classification of Temporal Bone Fractures
}

\author{
Sumit Sharma* \\ Department of ENT, Mayo Institute of Medical Sciences, Barabanki, India
}

Submission: March 01, 2019; Published: March 21, 2019

*Corresponding author: Sumit Sharma, Department of ENT, Mayo Institute of Medical Sciences, Barabanki, India

\section{Introduction}

The temporal bone is a very complex bone in the human body. It is also a very thick and hard structure located in the base of the skull. It houses many vital structures, including the cochlear and vestibular end organs, the facial nerve, the carotid artery, and the jugular vein. A temporal bone fracture can involve none or all of these structures. Associated trauma to other cranial nerves (other than the facial nerve; ie, VI [abducens], IX [glossopharyngeal], $\mathrm{X}$ [vagus] and XI [spinal accessory] can also cause paralysis [1]. The spectrum of temporal bone injury is extremely varied ranging from minor non-specific haematomas and laceration to more serious injuries like facial nerve paralysis (partial or complete), hearing loss (conductive, sensori-neural, or mixed), vertigo, dizziness, otorrhagia, cerebrospinal fluid (CSF) otorrhea, tympanic membrane perforation, and hemo-tympanum. Head injury is a major cause of Temporal bone fracture. Most cases of temporal bone fracture involve severe body and/or head trauma. In the adult population, approximately $90 \%$ of temporal bone fractures are associated with concurrent intracranial injuries and $9 \%$ with cervical spine injuries [2] hence its always important to asses all the injuries of the patient - grade them in severity and treat according to severity. However, the initial assessment of the temporal bone trauma is extremely important. Blunt trauma to the skull and penetrating trauma to the skull are the two major type of injury seen with trauma to the head. The nature of the injury will vary considerably based on the type of trauma delivered directly to the head.

\section{Etiopathogenesis}

An experimental study conducted by Matsui Tetal [3] for temporal bone fractures suggested that the forces necessary to produce the first fracture (seperations of the parietotemporal suture) are between $135 \mathrm{~kg}$ and $385 \mathrm{~kg}$. (average; $258 \mathrm{~kg}$ ) 2) while the forces necessary to produce the second fractures (true fractures) are between $195 \mathrm{~kg}$ and $550 \mathrm{~kg}$. (average; $356.3 \mathrm{~kg}$ ). They also suggested that the forces necessary to produce a fracture in the temporal region are much less than the forces necessary to do so in the frontal region. The ear is the most frequently damaged sense organ. It has been suggested that when head trauma is suf ficient to fracture the skull, 14 to $22 \%$ of injured patient sustain a temporal bone fracture. They occur across all age groups although $>70 \%$ fractures are seen in second, third, fourth decade. The Male: female ratio seen is 3:1, the predisposition in males is based on an increased number of males involved in high-risk activities compared with females, rather than on a weakness in their skull structures, and 8-to $29 \%$ of fractures of the temporal bone occur bilaterally [1].

The 3 major categories of injury to temporal include:

a) Blunt Injury - Most commonly seen in road traffic accidents, physical assault, and other impact injuries.

b) Penetrating Injury - Stab and gunshot wounds are the most common penetrating wounds. This also include injuries to the tympanic membrane by sharp objects like pencils, swab sticks etc.

c) Iatrogenic Injuries are mostly a result of mastoid surgery and most often either facial nerve is involved or the semicircular canals.

Children generally recover from temporal bone trauma with fewer complications than adults and experience a markedly lower incidence of facial nerve paralysis. Although temporal bone fractures in the pediatric population are also associated with a high incidence of intracranial complications [1].

\section{Classification of Temporal Bone fractures}

Up-till now two classification systems are available for temporal bone fractures as below:

a) Traditional (Based on the Fracture Line Radio-Logically)

i. Longitudinal

ii. Transverse

iii. Oblique

b) New Classification: (Based on the Involvement of Otic Capsule) 
i. Otic Capsule Sparing caused by a blow to the temporo-parietal region [4].

ii. Otic Capsule Involving here fracture runs directly into the otic capsule, damaging the cochlea and semicircular canals, and is caused by a blow to the occipital region [4].

Ulrich in 1926 [4] was the first to classify temporal bone fractures into longitudinal fractures and transverse fractures. Ghorayeb and Yeakley, in their study of 150 temporal bone fractures, found that the vast majority of fractures are actually oblique and quite often mixed. Ishman and Friedland [5] proposed a classification system that divides fractures into two groups: petrous and non-petrous. Petrous fractures extend into the petrous apex or the Otic capsule. Patients with petrous fracture are more likely to develop cerebrospinal leak and facial nerve injury. Non-petrous fractures involve neither the petrous apex nor the Otic capsule. However, they may extend into the middle ear or mastoid and are more likely to lead to conductive hearing loss.

\section{Traditional Classification}

The Longitudinal Fractures: These are the most common temporal bone fractures comprising about $80 \%$ of all the fractures [6]. Lateral impact injuries to the temporal bone are mostly responsible for these fractures - impacts involving the mastoid or the squamous temporal bone. The fracture line parallels the long axis of the petrous pyramid. It starts in the pars squamous (mastoid or external auditory canal) and extends through the postero-superior bony external canal, continues across the roof of the middle ear space anterior to the labyrinth, and ends antero-medially in the middle cranial fossa in close proximity to the foramen lacerum and ovale [6]. These fractures follow the path of least resistance, which often involves aerated regions, foramina, and suture lines. Longitudinal fractures most frequently traverse at some point through the middle ear [7]. The most common course of the fracture is anterior and extra-labyrinthine; however, although rare, intra-labyrinthine extension is possible. Again, bilateral temporal bone fractures are present in 8-29\% of all fractures, according to the medical literature.

Transverse Fractures: These comprise to about 20\% of all the Temporal bone fractures and are usually caused by antero-posterior (frontal or parietal) or postero-anterior (occipital) impact injuries to the skull. The fracture line runs at a right angle to the long axis of the petrous pyramid and starts in the middle cranial fossa (close to the foramen lacerum and spinosum). It then crosses the petrous pyramid transversely and ends at the foramen magnum. It may also extend through the internal auditory canal and injure the nerves directly [6]. The facial nerve is involved in $50 \%$ of cases and the injury site is anywhere from the internal auditory canal to the horizontal segment distal to the geniculate ganglion. Pneumo-labyrinth may be noted [8].

Oblique or Mixed Fractures: Sometimes fracture lines are neither Longitudinal nor Transverse or show a mixed pattern. These patterns, which extend both longitudinally and transverse- ly, are common. According to some authors, these patterns occur more often than isolated transverse or longitudinal fractures. A range of $62-90 \%$ of temporal bone fractures were designed as a mixed pattern in medical literature [6].

\section{New Classification}

The bony labyrinth (also the osseous labyrinth or Otic capsule) is the rigid, bony outer wall of the inner ear in the temporal bone. It consists of three parts: the vestibule, semicircular canals, and cochlea. These are cavities hollowed out of the substance of the bone and lined by periosteum. They contain a clear fluid, the perilymph, in which the membranous labyrinth is situated [9]. The fractures which do not involve the Otic capsule are called as the Otic capsule sparing fractures where as those which involve the Otic capsule are called as the Otic capsule involving fractures.

The Otic Capsule Sparing Fractures: They are more common of the two types accounting to about $95 \%$ of all the temporal bone injuries usually caused by Temporal or parietal trauma impact. The fracture line passes anterolateral to the Otic capsule involving Squamosa portion of temporal bone, postero-superior wall of the external auditory canal and tympanic membrane which is commonly involved and also the mastoid air cells and middle ear. The ossicular chain involvement is very common in this injury leading mostly to a conductive or mixed type of hearing impairment. Facial nerve involvement is less common whereas CSF leak if occurs is from the middle cranial fossa [1].

The Otic Capsule Involving Fractures: These are the rare type of fracture accounting about $5 \%$ of all the fractures and are usually due to occipital injuries. The fracture line passes through the Otic capsule involving the Foramen magnum, petrous pyramid, and Otic capsule, the jugular foramen, internal auditory canal, and foramen lacerum. The Tympanic membrane and external auditory canal not usually affected. Involvement of ossicular chain is very rare in this type, the hearing loss is Sensori-neural type and facial nerve involvement is very common. CSF leak if occurs, is from the posterior cranial fossa (middle ear, eustetian tube) [1]. Other other intracranial pathology (e.g. subdural or subarachnoid hemorrhage) are more common in this type [1]. A study conducted by Dahiya, Ravinderetal [10] suggested that compared with Otic capsule sparing fractures, patients with Otic capsule violating fractures were approximately two times more likely to develop facial paralysis, four times more likely to develop CSF leak, and seven times more likely to experience profound hearing loss, as well as more likely to sustain intracranial complications including epidural hematoma and subarachnoid hemorrhage.

The classification models indicated above are arbitrary but indeed useful to predict the pattern of injury. Most temporal bone fractures are completely irregular and not uniform in their pathway, following an oblique or mixed pattern as indicated above. Therefore, rather than the type of fracture, the evaluation of function is the critical issue and is mandatory. The presence or lack of a particular function indicates whether or not the cochlea, middle 
ear, vestibule, facial nerve, dura, or CNS is damaged, in spite of a demonstrated fracture or the lack thereof. The mentioned classifications are useful in predicting otologicsequelae but cannot predict neurological, neuro-ontological, or skull base complications [1]. The previous two classifications only focus on the injury to the petrous temporal bone and not much emphasis has been given to other parts of the temporal bone like the middle ear, mastoid etc. Injury to the facial nerve can be both intra-tympanic as well as in the petrous temporal bone and the management of the two is very different. It does not cover the injury to the tympanic membrane which is very common in temporal bone injury along with ossicular injury which has to be addressed by the specialists. A newer, simpler and more comprehensive classification is being suggested here which is very helpful in patient evaluation and treatment.

\section{Types of Temporal Bone Fractures}

i. Type 1. Involving the Squamous Part of Temporal Bone

ii. Type 2. Involving the Middle Ear Cleft

iii. Type 3. Involving the Pertous Temporal Bone

iv. Type 4. Mixed Type

These temporal bone fractures can further be classified as SAFE AND UNSAFE depending upon the presence or absence of the following complications:(involvement of vital structures leading to impaired functions):
a. Injury to Tympanic Membrane.
b. Injury to Ossicles.
c. Injury to Facial Nerve.
d. CSF Otorrhoea.
e. Injury to Cochlea and the Vestibule.
f. Injury to the Cochlear Nerve.
g. Intracranial Complications.
h. Unusual Complications of Temporal Bone Fractures (listed below)
i. Multiple - More Than One of the Above.
Unusual complications of temporal bone fractures [1].

I. Paralysis of Cranial Nerves IX, X and XI: These cranial nerves can be affected at the jugular foramen in petrous apex fractures.

II. Paralysis of Cranial Nerve VI: This condition usually occurs in the area of the Meckel cave and the Dorello canal.

III. Paralysis of Cranial Nerve V: This condition usually occurs in the area of Meckel cave.

IV. Intratemporal Carotid Artery Injury: This type of injury is rare however if a fracture of the carotid canal is noted using CT scanning, a carotid artery injury is a possibility.
V. Carotid Cavernous fistula is a delayed vascular complication of temporal bone fracture.

VI. Sigmoid Sinus Thrombosis: This condition occurs but is rare.

VII. Posttraumatic Cholesteatoma: This is a late complication of temporal bone fracture and is caused by skin entrapment in the cranial vault or temporal bone.

VIII.Classic Eagle Syndrome: Traumatic fracture of an ossified styloid and stylohyoid ligament can cause pressure on the external or internal carotid artery and pain may be referred to the cheek or eye, producing atypical pain.

IX. Sympathetic Cochleolabyrinthitis: Cochlear fracture may release inner ear antibodies and cause host sensitization resulting in initiation of autoimmune inner ear damage with development of autoantibodies directed against inner ear proteins, as seen in polyarteritisnodosa. This is a rare complication of temporal bone fracture. The condition is clinically significant because of the potential for hearing loss in the sole ear with hearing.

The isolated injury to squamous temporal bone, external auditory canal lacerations, temporal hematomas, can be considered safe as they do not require active interventions and resolve spontaneously. This classification is more helpful in patient management as a detailed information about each type of fracture is available, they can be easily diagnosed by routine investigations. It also helps in patient management as the safer fractures can be managed conservatively even by a non-specialist whereas the unsafe fractures require special precautions and specialist management (depending upon the type of injury) as some of them require special evaluation and even surgical intervention.

\section{Conclusion}

We have tried to analyze the various classifications available for the evaluation of temporal bone injury and found that the previous two classifications have their limitations as these injuries are more than injury to the Otic capsule or the petrous temporal bone, a more comprehensive classification is being presented here which covers all the aspects of the temporal bone injuries.

\section{References}

1. Antonio Riera March, Arlen D Meyers (2017) Temporal Bone Fractures.

2. Sun GH, Shoman NM, Samy RN, Cornelius RS, Koch BL, et al. (2011) Do contemporary temporal bone fracture classification systems reflect concurrent intracranial and cervical spine injuries? Laryngoscope 121(5): 929-932.

3. Matsui T, Kihira M, Kobayashi H (1975) Experimental studies of skull fracture in the temporal region. No ShinkeiGeka 3(2):123-129.

4. Stewart C Little, Bradley W Kesser (2006) Radiographic Classification of Temporal Bone Fractures: Clinical Predictability Using a New System. Arch Otolaryngol Head Neck Surg 132(12): 1300-1304.

5. Julio O Zayas, Yara Z Feliciano, Celene R Hadley, Angel A Gomez, et al. (2011) Temporal Bone Trauma and the Role of Multidetector CT in the Emergency department. Radiographics 31(6): 1741-1755. 
6. Kerr AG, Smyth GDL, Maran AGD, Stell PM (1979) Ear Trauma, Clinical Otolaryngology. Blackwell Scientific Publications 44(2): 113-114.

7. Ana Maria DoffémondCostaI, Juliana Oggioni GaiottiI, Caroline Laurita Batista CoutoI, Renata Lopes Furletti Caldeira Diniz, Emília Guerra Pinto Coelho Motta, et al. (2013) Temporal bone trauma and complications: computed tomography findings. Radiol Bras 46(2).

8. Choi HG, Lee HJ, Lee JS, Kim DH, Hong SK, et al. (2015) The Rates and Clinical Characteristics of Pneumolabyrinth in Temporal Bone Fracture. Otol Neurotol 36(6): 1048-1053.
9. Little SC Kesser, BW (2006) Radiographic classification of temporal bone fractures: Clinical predictability using a new system. Archives of Otolaryngology-Head \& Neck Surgery 132(12): 1300-1304.

10. Dahiya R, Keller JD, Litofsky NS, Bankey PE, Bonassar LJ, et al. (1999) Temporal Bone Fractures: Otic Capsule Sparing versus Otic Capsule Violating Clinical and Radiographic Considerations. J Trauma 47(6): 1079-1083.

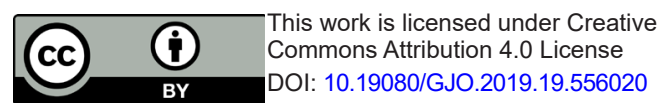

\section{Your next submission with Juniper Publishers will reach you the below assets}

- Quality Editorial service

- Swift Peer Review

- Reprints availability

- E-prints Service

- Manuscript Podcast for convenient understanding

- Global attainment for your research

- Manuscript accessibility in different formats

( Pdf, E-pub, Full Text, Audio)

- Unceasing customer service

Track the below URL for one-step submission https://juniperpublishers.com/online-submission.php 